\title{
Percutaneous kyphoplasty for the treatment of spinal metastases
}

\author{
FENG CHEN $^{1}$, YONG-HUI XIA ${ }^{1}$, WEN-ZHEN CAO $^{2}$, WEI SHAN $^{1}$, YANG GAO $^{1}$, BO FENG $^{1}$ and DIFEI WANG ${ }^{3}$ \\ ${ }^{1}$ Department of Interventional Radiology, The First Affiliated Hospital of China Medical University, Shenyang, \\ Liaoning 110001; ${ }^{2}$ Department of Intensive Care Unit Department, Qianfoshan Hospital Affiliated to Shandong University \\ Jinan, Shandong 250014; ${ }^{3}$ Department of Geriatrics, The First Affiliated Hospital of China Medical University, \\ Shenyang, Liaoning 110001, P.R. China
}

Received November 16, 2014; Accepted November 27, 2015

DOI: $10.3892 / \mathrm{ol} .2016 .4121$

\begin{abstract}
The bones are the most common location for metastases, which may cause severe pain and damage, including osteolytic destruction and fractures. Pathological fractures of the spine are extremely painful and cause significant disability and morbidity in patients. Traditional open surgery has numerous complications, and radiation therapy may take weeks to become effective. To avoid the trauma and complication of open surgery, percutaneous kyphoplasty (PKP) is a minimally invasive procedure that has played a great role in the treatment of spinal metastases over the past several years. To evaluate the efficacy and safety of the treatment of spinal metastasis using PKP, the present study evaluated 282 patients who had received PKP between April 2009 and June 2014. The efficacy of PKP was evaluated using the visual analog scale for pain (VAS), Karnofsky performance score (KPS) and quality of life (QOL) score (short form with 36 questions). The KPS and QOL were measured pre-operatively and 3 months post-operatively. In addition, radiographical data, including the degree of restoration of the kyphotic angle and the anterior vertebral height, and leakage of bone cement, were measured. The safety of the surgery was assessed by complications and side effects reported during or subsequent to surgery. The present study measured the parameters prior to the surgery and at $24 \mathrm{~h}, 3$ months, 6 months and 1 year post-surgery, as well as at the last follow-up date. The range of the follow-up time was between 105 days and 15 months (mean, 401 days). The 282 patients
\end{abstract}

Correspondence to: Professor Bo Feng, Department of Interventional Radiology, The First Affiliated Hospital of China Medical University, 155 Nanjing North Street, Shenyang, Liaoning 110001, P.R. China

E-mail: fb6772@sina.com

Professor Difei Wang, Department of Geriatrics, The First Affiliated Hospital of China Medical University, 155 Nanjing North Street, Shenyang, Liaoning 110001, P.R. China

E-mail: wdf81m@163.com

Key words: spinal metastases, minimally invasive, percutaneous kyphoplasty, bone cement underwent successful operations and the pain felt by the patients prior to the surgery was significantly alleviated. In addition, the analgesic intake of patients greatly decreased following PKP. KPS improved prior to and 3 months after the surgery. QOL also improved prior to and 3 months after the surgery. Radiographical data demonstrated that the kyphotic angle decreased following PKP, and the anterior vertebral height increased. Paravertebral leakage of bone cement occurred in 10 patients through a cortical defect, but without spinal cord compression or pulmonary embolism. Therefore, as a minimally invasive procedure, PKP may not only rapidly relieve the pain and disability experienced by patients, but it may also restore the kyphotic angle observed at the 1-year follow-up. Notably, PKP may safely improve the QOL of patients.

\section{Introduction}

The most frequent site of bone metastasis is the spine, and $39 \%$ of patients with bone metastases exhibit axial bone lesions $(1,2)$. The most common location for spinal metastases is the thoracic spine (40-50\%); however, all spinal column levels may be affected and $>70 \%$ of spinal metastases may involve more than one vertebral level at presentation (3). The predominant symptom in spinal metastases is pain, including constant localized, radicular and axial pain (4). In addition to pain, spinal metastases may lead to pathological fractures of the vertebrae and paraplegia caused by spinal cord compression (5). Advances in diagnosing and treating patients with spinal metastases have improved the survival time and quality of life (QOL) of patients, therefore, controlling the pain that is experienced has become a major focus (6). Conservative treatments, including the use of analgesics, chemotherapy and radiotherapy, can be transient and ineffective, and traditional invasive surgery is typically unsuitable for patients with spinal metastases due to the high risk of surgical complications and the associated short life expectancy. More recent technological advances in combination with innovative interventional radiology techniques have provided less invasive treatment therapy for patients with spinal metastases. Percutaneous kyphoplasty (PKP) is used to treat patients with painful metastases in the spine (7). PKP is a minimally invasive, radiologically-guided procedure that involves injecting bone cement into target vertebrae following the insertion of a 
balloon into the vertebral body to create a cavity. This allows relatively low pressure and high-viscosity bone cement disposition, which lowers the cement leakage rate (8-10).

The current study retrospectively reviewed the clinical and radiological outcomes of PKP in patients with osteolytic-associated spinal metastases in the thoracic or lumbar vertebrae, with an aim to evaluate the efficacy and safety of PKP in the treatment of these patients.

\section{Patients and methods}

Patient characteristics. Between April 2009 and June 2014, 282 patients with 399 vertebral lesions (thoracic vertebrae, 223; lumbar vertebrae, 176) received PKP at the First Affiliated Hospital of China Medical University (Shenyang, China) in our hospital. The majority of the patients received systemic chemotherapy or radiotherapy prior to PKP; however, the severe pain these patients experienced remained following the treatments. All the patients met the following inclusion criteria: Presence of a primary malignant tumor, which was diagnosed by pathology or cytology; grade D-E spinal cord function, according to the Frankel grading system (11); adequate hematological, hepatic, renal, neuronal and cardiac functions, and the ability to maintain a prone position for $\geq 2 \mathrm{~h}$; a Karnofsky performance score (KPS) of $\geq 60$; an expected survival time of $\geq 3$ months; vertebral destruction, which was dominated by osteolytic lesions; thoracic and lumbar vertebral fractures confirmed by physical and imaging examinations; and a lesion region located below the 6th thoracic vertebra. The characteristics and tumor data of the patients are presented in Table I. All patients were informed of the future publication of this study and provided their consent. The study was approved by the Institutional Board at the First Affiliated Hospital of China Medical University.

Evaluating the efficacy of PKP. The efficacy of PKP was evaluated using the visual analog scale for pain (VAS), KPS and QOL scores [short form with 36 questions (SF-36)]. In addition, radiographical data, including the degree of restoration of the kyphotic angle and anterior vertebral height, and the leakage of bone cement, were measured. The safety of PKP was assessed by evaluating the complications and side effects reported during or post-surgery. A clinical assessment of the patients was performed on the day prior to the surgery and at $24 \mathrm{~h}, 3$ months, 6 months and 1-year post-surgery, as well as at the last follow-up date. The KPS and QOL scores were calculated 3 months after PKP, and baseline KPS and QOL scores were assessed prior to the procedure. A clinical follow-up examination of the patients was conducted by Dr Feng Chen, Dr Wei Shan and Dr Yang Gao (Department of Interventional Radiology, First Hospital of China Medical University), and diagnostic images were independently evaluated by Mr. Xiang Wang (Department of Geriatrics, The First Affiliated Hospital of China Medical University). All the patients received conventional chemotherapy following the surgery, which was specific to the primary tumor.

PKP procedure. The following instruments were used in the PKP procedure: Puncture needles (3.2-4.0 $\mathrm{mm}$ in diameter), hollow diamond and solid diamond tipped needles, precision
Table I. Clinical characteristics of 282 patients with spinal metastases.

\begin{tabular}{lc}
\hline Characteristics & Value \\
\hline Age in years, $\mathrm{n}(\%)$ & \\
$25-34$ & $11(3.90)$ \\
$35-44$ & $12(4.26)$ \\
$45-54$ & $84(29.79)$ \\
$55-64$ & $60(21.28)$ \\
$65-74$ & $96(34.04)$ \\
$75-84$ & $12(4.26)$ \\
$85-94$ & $7(2.48)$ \\
Gender, $\mathrm{n}(\%)$ & \\
Female & $156(55.32)$ \\
Male & $126(44.68)$ \\
Primary tumor, $\mathrm{n}(\%)$ & \\
Liver & $60(21.28)$ \\
Lung & $132(46.81)$ \\
Breast & $24(8.51)$ \\
Cervical & $24(8.51)$ \\
Prostate & $13(4.61)$ \\
Kidney & $12(4.26)$ \\
Bladder & $12(4.26)$ \\
Lymph & $5(1.77)$ \\
No. of vertebral lesions treated, $\mathrm{n}(\%)$ & \\
1 & $180(63.83)$ \\
2 & $84(29.79)$ \\
3 & $14(4.96)$ \\
4 & $4(1.42)$ \\
VAS & $6.85 \pm 0.97$ \\
KPS & $70.42 \pm 10.83$ \\
QOL & $67.74 \pm 9.33$ \\
Kyphotic angle, & $16.68 \pm 1.46$ \\
Anterior vertebral height, mm & $18.45 \pm 5.62$ \\
\hline & \\
\hline & \\
\hline &
\end{tabular}

VAS, KPS, QOL, kyphotic angle and anterior vertebral height are presented as the mean \pm standard deviation. VAS, pain visual analogue scale; KPS, Karnofsky performance score; QOL, quality of life score.

injector pressure device (Longguan Co., Jinan, China) to assess cement pressure during injection, surgical balloon, bone cement injection gun (Longguan Co.); bone cement (Heraeus Medical GmbH, Wehrheim, Germany); and surgical hammer (Beijing Operation Apparatus Factory, Beijing, China).

$\mathrm{X}$-ray, computed tomography $(\mathrm{CT})$ and magnetic resonance imaging (MRI) were performed prior to surgery to determine the location and number of lesions, and the degree of collapse of the vertebrae (Fig. 1). In addition, CT and MRI established if the back wall of the vertebral body was intact and if the spinal cord was compressed. PKP was guided by a Digital Subtraction Angiology (DSA) machine (GE Healthcare Bio-Sciences, Pittsburgh, PA, USA) in the interventional radiology suite following an analysis of the radiological 

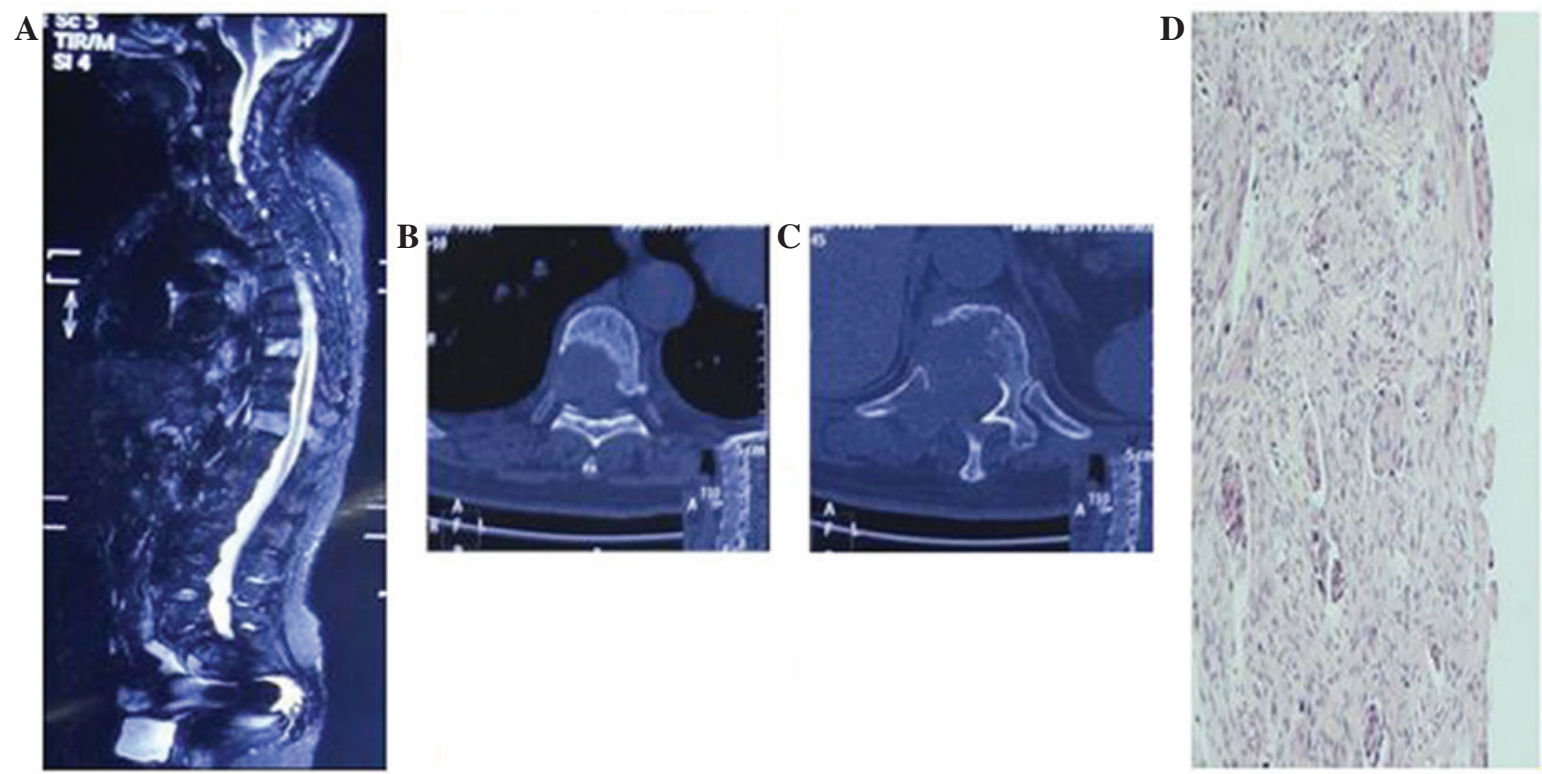

Figure 1. Computed tomography and magnetic resonance imaging were performed prior to surgery to determine the location and number of lesions present, and the degree of collapse of the vertebra. (A) T8 and 11 with metastases from lung cancer, exhibiting a high signal on short inversion time inversion recovery. (B) T8 and (C) T11 exhibiting osteolytic damage. (D) Image showing the pathological type of the primary tumor as squamous cell carcinoma (stain, p63; magnification, $\mathrm{x} 100)$. $\mathrm{T}$, thoracic vertebra.
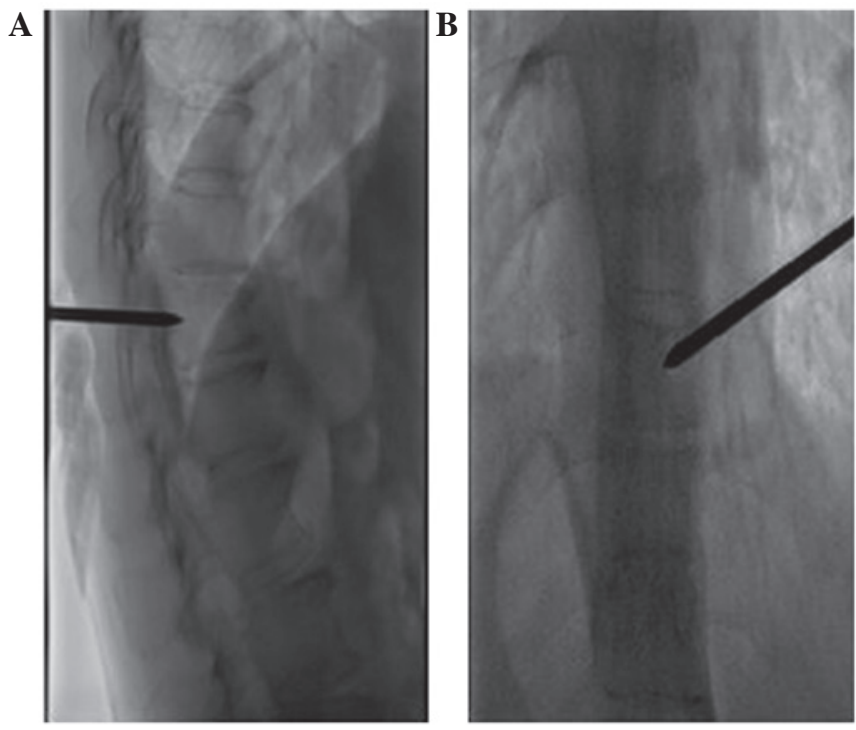

Figure 2. Intra-operative images revealing the injection needle puncturing the vertebra. (A) The tip of the injection needle reaches the posterior margin of the vertebral body in the lateral view. (B) The tip of the injection needle reaches the pedicle medial margin.

images. Local anesthesia ( $2 \%$ lidocaine; $5 \mathrm{ml}$ ) was administered to the patient prior to the surgery. The patient was placed in the prone position and injection needles were used to puncture unilaterally or bilaterally through the pedicle of the vertebral arch (if the metastasis was located in a lumbar vertebra) or rib vertebral joints (if the metastasis was located in a thoracic vertebra).

When the needle tip reached the back wall of the vertebra (Fig. 2), 3-dimensional CT scanning was used, in combination with DSA, to ensure that the spinal cord was not punctured and the procedure was effective (Fig. 3). A surgical hammer

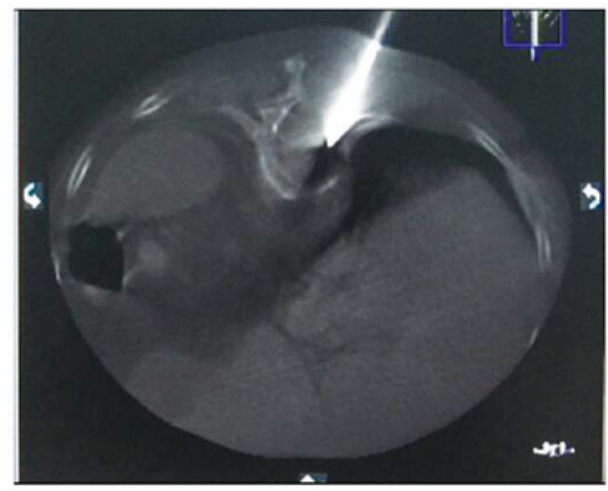

Figure 3. A computed tomography scan revealing the injection needle puncturing the vertebra through the pedicle without touching the spinal cord.

was used to aid the needle placement into the anterior quadrant of the targeted vertebral body. Hollow and solid diamond tipped needles were used to polish the tract. Subsequently, a balloon, which was used to restore vertebral body height and create a cavity in the vertebra for the injection of bone cement, was inserted into the vertebra using fluoroscopic monitoring. The balloon was inflated and viewed using a contrast agent. If it was challenging to inflate the balloon or a portion of the balloon reached the vertebral margin, inflation was terminated and the balloon was removed (Fig. 4). Bone cement, a mixture of polymethyl methacrylate (PMMA) and non-ionic contrast medium, was injected into the vertebra using a bone cement injecting gun and fluoroscopic monitoring. When the PMMA had solidified the injection needles were removed. The volume of bone cement injected into the vertebra was $1-5 \mathrm{ml}$, with a mean of $1.9 \mathrm{ml}$ for the thoracic vertebrae and $1.97 \mathrm{ml}$ for the lumbar vertebrae (Figs. 5 and 6). Following the surgery, the patients were required to rest for $6 \mathrm{~h}$, and during this period the vital signs, neurology, urine volume, 
A

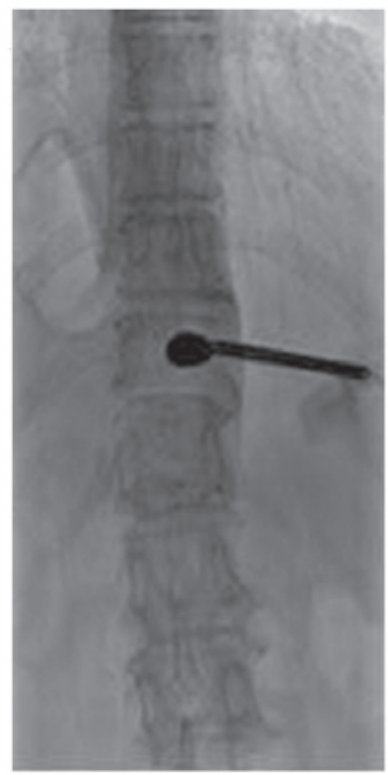

B

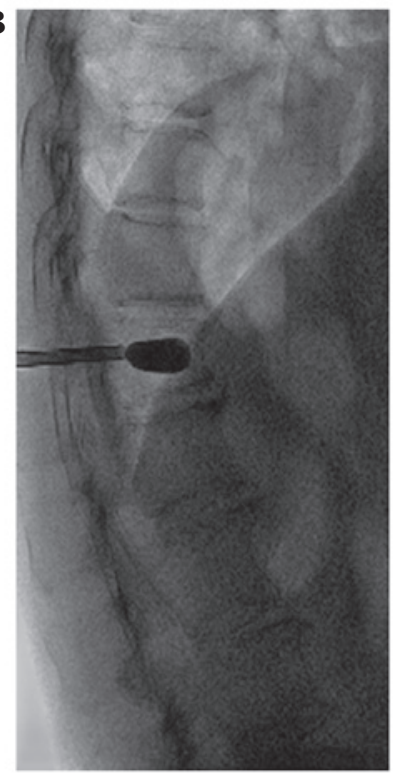

Figure 4. A surgical balloon was used to create a cavity in the vertebra and restore the height of the vertebral body, as shown in the (A) posterior and (B) lateral view.

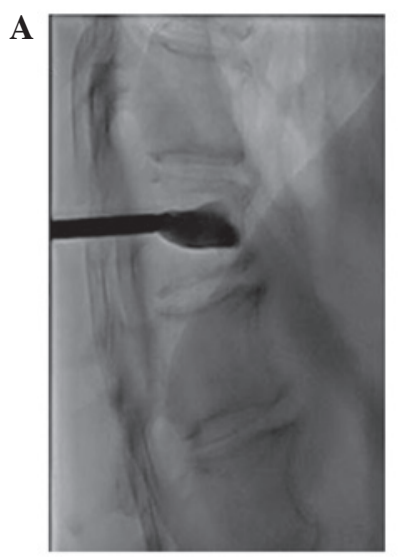

B
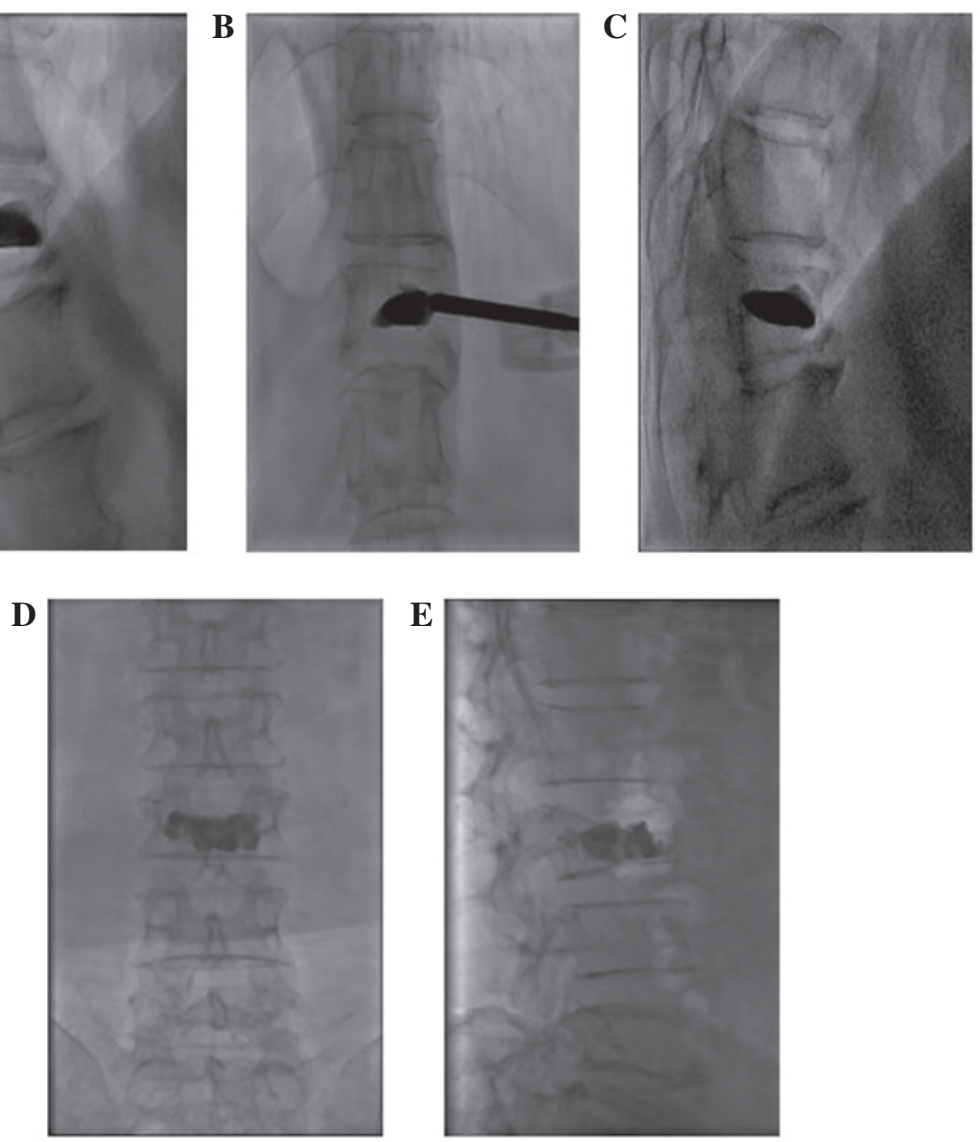

$\mathbf{E}$

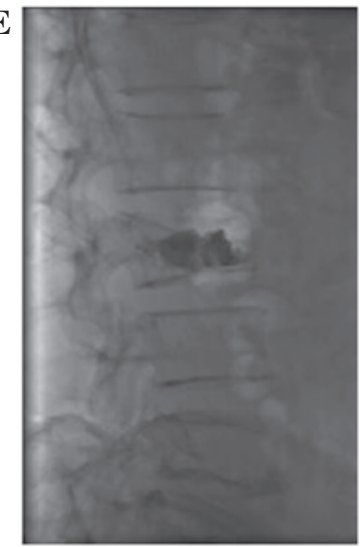

Figure 5. (A and B) The injection of bone cement into the vertebra using a bone cement injection gun. (C) Even post-operative spread of the bone cement in the cavity, which the surgical balloon created. (D and E) Bone cement filling the entire area of lesion destruction [(D) posterior view; (E) lateral view].

bowel movement, sensory motor functions of the lower limbs, and alterations in routine blood tests and $\mathrm{CD}^{+}$and $\mathrm{CD} 8^{+}$ $\mathrm{T}$ cell subpopulations were monitored. The patients received mannitol (250 ml injection, daily) and solu-medrol (80 mg injection, daily) for three days to treat spinal cord edema.
Statistical analysis. Continuous data were expressed as the mean \pm standard deviation (SD). A comparison of continuous variables pre- and post-surgery was performed using a one-way analysis of variance. $\mathrm{P} \leq 0.05$ was considered to indicate a statistically significant difference. 

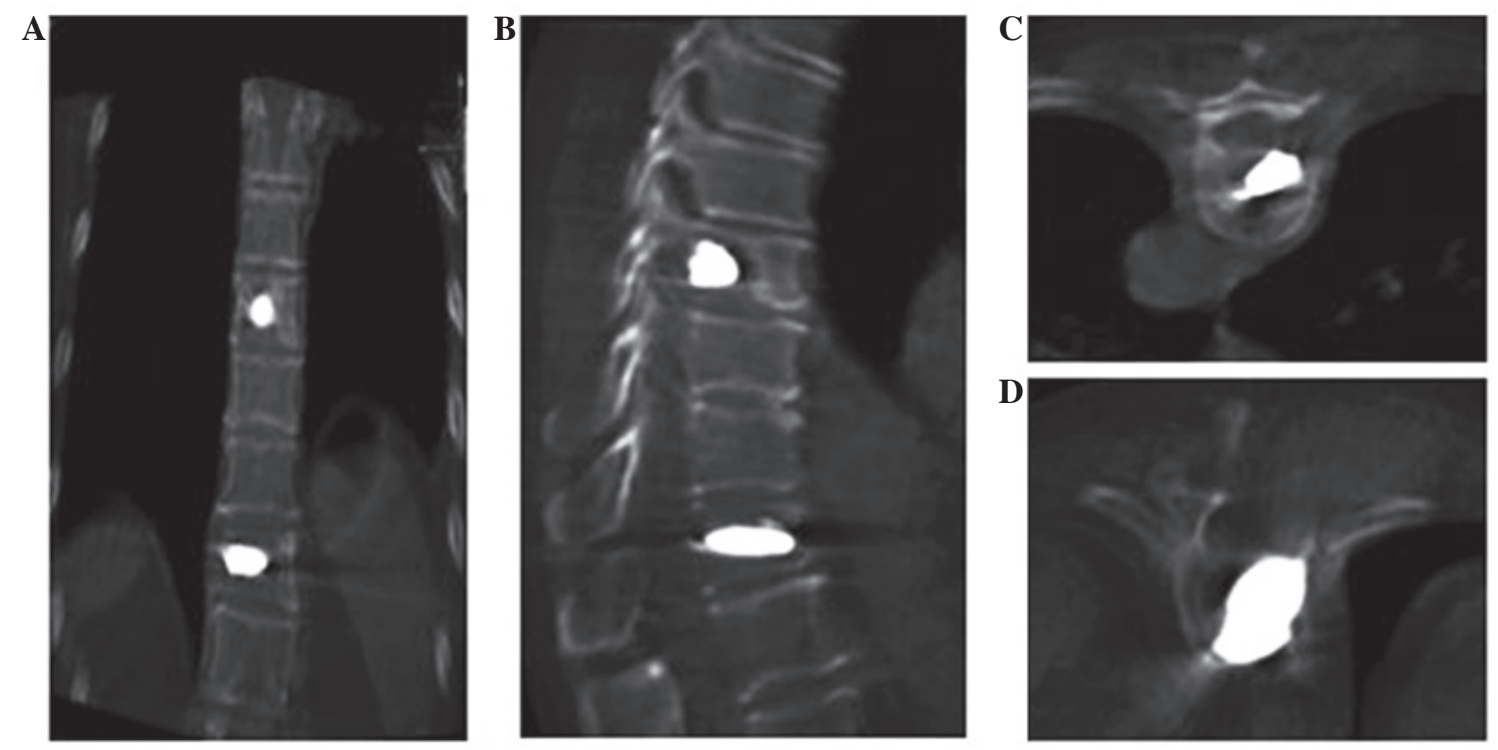

Figure 6. Computed tomography scans post-surgery confirming that the bone cement had spread safely through the vertebra without extravasation or nerve compression. (A) Coronal view of T8 vertebra; (B) lateral view of T11 vertebra; (C) cross-sectional view of T8 vertebra; (D) cross-sectional view of T11 vertebra.
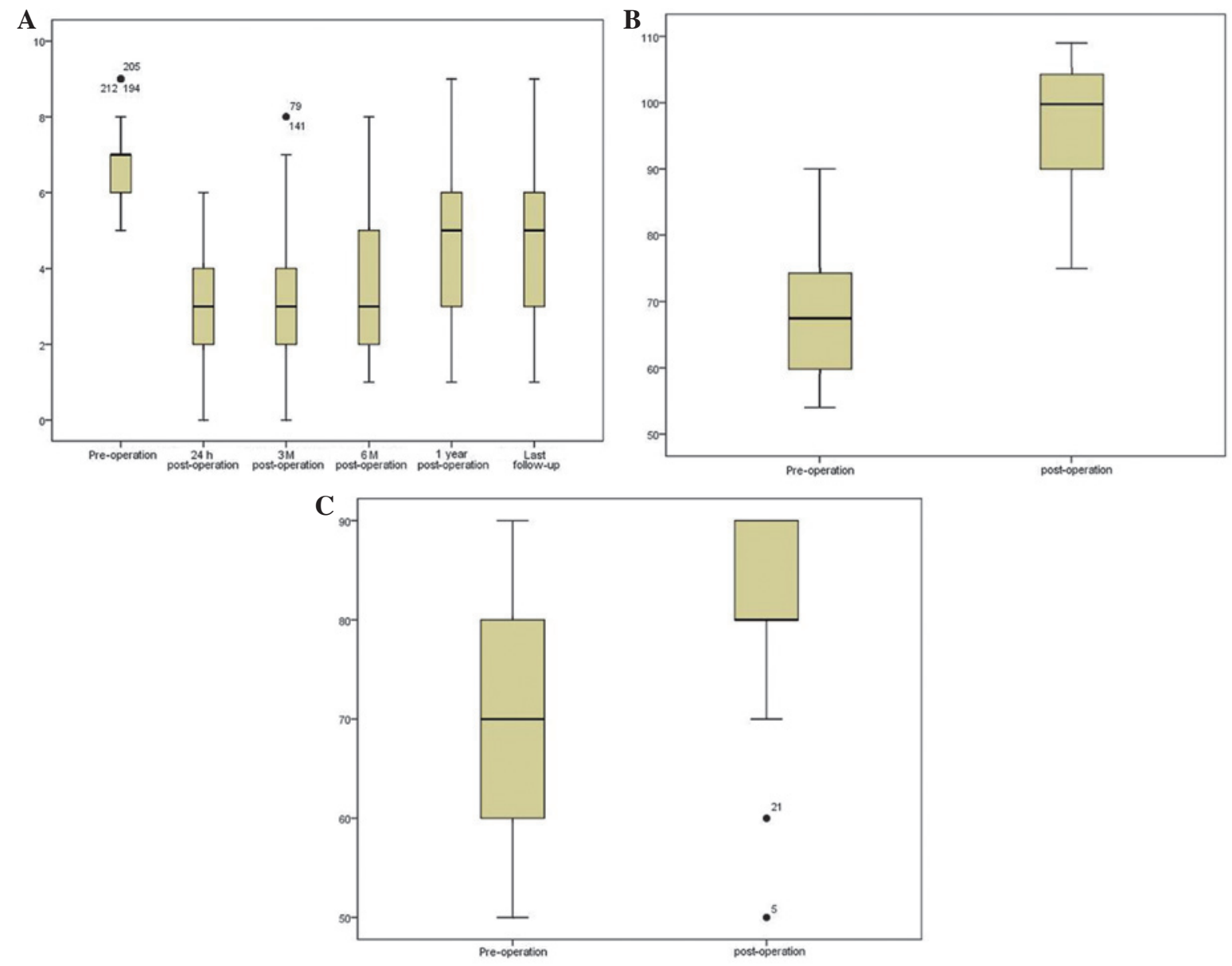

Figure 7. Percutaneous kyphoplasty efficacy evaluation. The (A) visual analog scale for pain, (B) Karnofsky performance score and (C) quality of life score were all ameliorated post-surgery compared with prior to surgery.

\section{Results}

Patient follow-up. The PKP procedures performed on 282 patients with spinal metastases were successful and without severe complications. The success rate of the puncture surgery was $100 \%$. The clinical assessment of the patients was performed at $24 \mathrm{~h}, 3$ months, 6 months and 1 year post-surgery, and the last follow-up assessment 

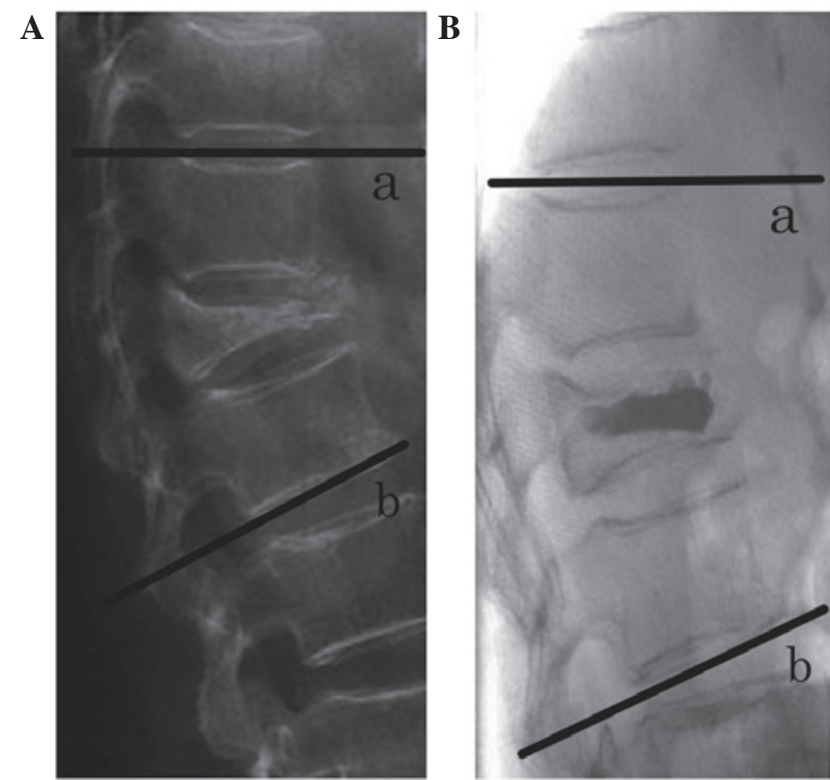

Figure 8. Kyphotic angle defined as the Cobb angle measured between the superior endplate of (a) the vertebra one level above the treated vertebra to the inferior endplate of (b) the vertebra one level below the treated vertebra, using lateral X-ray images. (A) Lateral view pre-PKP; (B) lateral view post-PKP. PKP, percutaneous kyphoplasty.

was performed via outpatient review or telephone. All 282 patients underwent follow-up assessments $24 \mathrm{~h}$ and 3 months post-surgery, and 272 patients were evaluated at 6 months post-surgery. A total of 60 patients were lost to follow-up at 1-year post-surgery. The follow-up duration ranged between 105 days and 15 months (mean, 401 days).

$V A S$. For pain evaluation, the patients were asked to evaluate their degree of pain, according to VAS, which uses a score of 0-10 to indicate levels of pain between mild and severe ( 0 , no pain; 10 , severe pain). All the patients exhibited pain relief following the surgery. The pre-operative VAS was $6.85 \pm 0.97$, which decreased to $3.11 \pm 1.3824 \mathrm{~h}$ post-operatively $(\mathrm{P}<0.001)$. The VAS of the patients remained largely unaltered at 3 months $(3.17 \pm 1.521 ; \mathrm{P}<0.001 ; 282$ patients $)$, 6 months $(3.58 \pm 1.63 ; \mathrm{P}<0.001 ; 272$ patients $)$ and 1 year (4.61 $\pm 1.65 ; \mathrm{P}<0.001 ; 222$ patients), and at the last follow-up (4.91 $\pm 1.99 ; \mathrm{P}<0.001 ; 219$ patients) (Fig. 7A). In addition, the analgesic intake of the patients decreased following PKP (data not shown).

$K P S$. The physical condition of the patients was assessed by KPS. KPS score is used to assess how the disease of a patient is progressing, how it affects the daily living abilities of the patient, and determines the appropriate treatment and prognosis of the patient. A patient with a KPS score of 100 , exhibits no signs and symptoms, while a score of 0 indicates patient mortality. Thus, the higher the score, the better the health status. In the present study, the pre-operative KPS was $70.42 \pm 10.83$, which increased at 3 months post-surgery

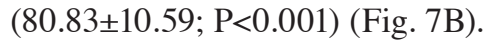

QOL. QOL (SF-36) was developed by a USA Boston health study $(12,13)$, and was based on a previous study concerning the medical outcomes (14). The QOL health questionnaire is widely used in the evaluation of clinical trials and health policies. The questionnaire consists of 9 topics: Physical functioning, role-physical, bodily pain, general health, vitality, social functioning, role-emotional, mental health and reported health transition. A patient with a score of 100 , exhibits no signs and symptoms, while a score of 0 indicates patient mortality. Thus, the higher the score, the better the health status. In the present results, the pre-operative QOL score was $67.74 \pm 9.33$, which then increased to $97.41 \pm 9.55$ at 3 months post-surgery ( $\mathrm{P}<0.001$; Fig. 7C).

Radiographical evaluation. The kyphotic angle is defined as the Cobb angle measured between the superior endplate of the vertebra one level above the treated vertebra and the inferior endplate of the vertebral body one level below the treated vertebra, using a lateral X-ray image (Fig. 8) (15). In the present study, the pre-operative kyphotic angle was $15.68 \pm 1.46^{\circ}$, which decreased at 3 months post-surgery $\left(7.71 \pm 1.51^{\circ}\right)(\mathrm{P}<0.05)$. In addition, the pre-operative anterior vertebral height $(18.40 \pm 5.59 \mathrm{~mm})$ increased to $24.20 \pm 6.261 \mathrm{~mm}$ at 3 months post-surgery $(\mathrm{P}<0.05)$.

Complications. Complications reported consisted of minor paravertebral leakage of bone cement, which occurred in 10 patients due to a cortical defect. Spinal cord compression, pulmonary embolism, leukopenia, decreased immunological function and nerve root compression were not detected in any patient.

\section{Discussion}

Intractable pain is a common symptom of bone metastases (16). Even following the administration of analgesics and chemotherapy, the alleviation of pain may be challenging (17). This leads to a poor QOL and decreased survival time of patients with bone metastases. The majority of treatment is palliative (18). Surgery and radiation therapy or a combination of the two may be used, but often, such patients with a limited life expectancy do not undergo surgery due to the long recovery period, and high morbidity and mortality rates that may result from surgery (19). Surgery is not suitable for treating patients with multiple metastatic spinal tumors (19-21). Radiotherapy is the most usual treatment for spinal metastases; however, pain relief is delayed and not always absolute (22). Therefore, PKP, which is a minimally invasive technique, is a potential treatment option for patients with spinal metastases, and is currently used for pain relief and spinal stabilization. Due to the development of interventional radiology, PKP has become widely accepted as an effective treatment option for patients with spinal metastases (23).

Cotten et al (24) and Cortet et al (25) reported that percutaneous vertebroplasty plays a great role in treating patients with vertebral metastases and multiple myeloma, and that the pain patients experience could completely disappear. The remission rate of the vertebral metastases was 67.5 and $68.5 \%$, respectively, and the partial pain relief rates were each $30 \%$. The majority of lesions were treated by filling the lesion with bone cement, which stagnates disease and provides structure 
to the spine, preventing additional damage and collapse. By contrast, PKP, attempts to restore spinal alignment by the placement of bone cement at a lower pressure into a cavity in the vertebrae, which is created using a surgical balloon (26). Previous studies have reported that PKP results in considerably less cement extravasation compared with injecting bone cement into vertebral bodies at a higher pressure or without balloon insertion (9,27-29).

In this study, the mean VAS score decreased significantly at 6 months and the last follow-up (mean \pm SD: $3.58 \pm 1.63$ and $4.91 \pm 1.99$, respectively). Therefore, the patients in the study exhibited a significant improvement in pain relief following surgery, which may be associated with the effect of the bone cement and the stabilization of the spine (26). The dependence that the patients had on analgesics also greatly decreased. In addition, the functional status of the patients increased. The mean pre-operative KPS score increased between $70.42 \pm 10.83$ and $80.83 \pm 10.60$ post-operatively. The current results demonstrated that the insertion of the surgical balloon restored the pre-operative mean height of the vertebra, between $18.40 \pm 5.59$ and $24.20 \pm 6.26 \mathrm{~mm}$ post-operatively. Furthermore, the mean kyphotic angle was reduced from $15.68 \pm 1.461^{\circ}$ pre-operation to $7.71 \pm 1.512^{\circ}$ post-operation. A mean correction of $7.9^{\circ}$ was achieved in the local spinal kyphosis, which is similar to the $8.8^{\circ}$ reported by a previous study (29).

The most common complication of PKP is extravasation of the bone cement $(30,31)$. A previous meta-analysis demonstrated cement extravasation in $7 \%$ of patients following PKP and $20 \%$ following vertebroplasty (32). In the present study, cement extravasation occurred asymptomatically in $11.5 \%$ of the vertebrae treated. The cavity formation in PKP significantly decreases the rate of vascular and transcortical extravasation of the cement (29). Compared with previous studies, this finding indicated that an injection of high-viscosity cement at a low pressure into a previously formed cavity (PKP) is more effective than an injection of low-viscosity cement at high pressure into an unreduced vertebral body (vertebroplasty) (33-35). Therefore, PKP is considered to be a safer and more effective treatment option.

There were a few limitations to the present study. Firstly, there were a small number of patients, which possibly affected the statistical power. Secondly, the study had a short follow-up period (mean, 401 days), which is considered to be too short a time period. Thirdly, the present study was retrospective, although comprehensive data analysis was performed. Therefore, additional prospective studies may aid in gathering more definitive evidence.

In conclusion, the present study indicates that PKP is an effective and minimally invasive procedure for the treatment of patients with spinal metastases, and that the technique leads to a significant improvement in the pain and functional status of patients. In addition, PKP is more manageable and carries less risk compared with vertebroplasty.

\section{References}

1. Berrettoni BA and Carter JR: Mechanisms of cancer metastasis to bone. J Bone Joint Surg Am 68: 308-312, 1986.

2. Tatsui H, Onomura T, Morishita S, Oketa M and Inoue T: Survival rates of patients with metastatic spinal cancer after scintigraphic detection of abnormal radioactive accumulation. Spine 21 2143-2148, 1996.
3. Chi JH and Gokaslan ZL: Vertebroplasty and kyphoplasty for spinal metastases. Curr Opin Support Palliat Care 2: 9-13, 2008.

4. Georgy BA: Metastatic spinal lesions: State-of-the-art treatment options and future trends. AJNR Am J Neuroradiol 29: 1605-1611, 2008.

5. Yang ZZ, Xu JB, Yuan T, Qian BS, Zhang JY, Li WZ, Li JL, Xiao YB, Peng M, Li Y and Luan L: Treating metastatic vertebral tumor with percutaneous vertebroplasty: A report of 28 cases. Ai Zheng 24: 194-198, 2005 (In Chinese).

6. Zhang HT, Chen GD, Yang HL and Luo ZP: Percutaneous kyphoplasty in the treatment of osteoblastic-related spinal metastases. J Spinal Disord Tech: Jul 26, 2013 (Epub ahead of print).

7. Qian Z, Sun Z, Yang H, Gu Y, Chen K and Wu G: Kyphoplasty for the treatment of malignant vertebral compression fractures caused by metastases. J Clin Neurosci 18: 763-767, 2011.

8. Dudeney S, Lieberman IH, Reinhardt MK and Hussein M: Kyphoplasty in the treatment of osteolytic vertebral compression fractures as a result of multiple myeloma. J Clin Oncol 20: 2382-2387, 2002

9. Lieberman IH, Dudeney S, Reinhardt MK and Bell G: Initial outcome and efficacy of 'kyphoplasty' in the treatment of painful osteoporotic vertebral compression fractures. Spine 26: 1631-1638, 2001.

10. Bouza C, López T, Magro A, Navalpotro L and Amate JM: Efficacy and safety of balloon kyphoplasty in the treatment of vertebral compression fractures: A systematic review. Eur Spine J 15: 1050-1067, 2006

11. Frankel HL, Hancock DO, Hyslop G, Melzak J, Michaelis LS, Ungar GH, Vernon JD and Walsh JJ: The value of postural reduction in the initial management of closed injuries of the spine with paraplegia and tetraplegia. I. Paraplegia 7: 179-192, 1969.

12. Ware JE, Snow KK, Kosinski M and Gandek B: SF-36 Health Survey: Manual and Interpretation Guide. The Health Institute, New England Medical Center, Boston, MA, 1993.

13. Newnham EA, Harwood KE and Page AC: Evaluating the clinical significance of responses by psychiatric inpatients to the mental health subscales of the SF-36. J Affective Disord 98: 91-97, 2007.

14. Bai GT, Ma YW and Jiang L: Application progress of SF-36 at home and abroad. J Clin Res 12: 2367-2368, 2009 (In Chinese).

15. Cobb J: Outline for the study of scoliosis. AAOS Instr Course Lect 5: 261-275, 1948.

16. Bienz M and Saad F: Management of bone metastases in prostate cancer: A review. Curr Opin Support Palliat Care 9: 261-267, 2015.

17. Silverman SL: The clinical consequences of vertebral compression fracture. Bone 13 (Suppl 2):S27-S31, 1992.

18. McDonald R, Chow E, Rowbottom L, Bedard G, Lam H, Wong E, Popovic M, Pulenzas N and Tsao M: Quality of life after palliative radiotherapy in bone metastases: A literature review. J Bone Oncol 4: 24-31, 2014.

19. Yang Z, Yang D, Xie L, Sun Y, Huang Y, Sun H, Liu P and Wu Z: Treatment of metastatic spinal tumors by percutaneous vertebroplasty versus percutaneous vertebroplasty combined with interstitial implantation of 125I seeds. Acta Radiol 50: 1142-1148, 2009.

20. Li Y, Gu YF, Sun ZK, Wu CG, Li YD, Wang W, Chen YC and $\mathrm{Lu} \mathrm{J}$ : Comparison of percutaneous vertebroplasty with and without interventional tumour removal for malignant vertebral compression fractures with symptoms of neurological compression. Eur Radiol 23: 2754-2763, 2013.

21. Taylor JW and Schiff D: Metastatic epidural spinal cord compression. Semin Neurol 30: 245-253, 2010.

22. Shimony JS, Gilula LA, Zeller AJ and Brown DB: Percutaneous vertebroplasty for malignant compression fractures with epidural involvement. Radiology 232: 846-853, 2004.

23. Sun G, Jin P, Li M, et al: Percutaneous vertebroplasty for pain management in spinal metastasis with epidural involvement. Technol Cancer Res Treat 10: 267-274, 2011.

24. Cotten A, Dewatre F, Cortet B, Assaker R, Leblond D, Duquesnoy B, Chastanet P and Clarisse J: Percutaneous vertebroplasty for osteolytic metastases and myeloma: Effects of the percentage of lesion filling and the leakage of methyl methacrylate at clinical follow-up. Radiology 200: 525-530, 1996.

25. Cortet B, Cotton A, Boutry N, Dewatre F, Flipo RM, Duquesnoy B, Chastanet $\mathrm{P}$ and Delcambre B: Percutaneous vertebroplasty in patients with osteolytic metastases or multiple myeloma. Rev Rev Rhum Engl Ed 64: 177-183, 1997.

26. Yu C-W, Hsieh M-K, Chen LH, et al: Percutaneous balloon kyphoplasty for the treatment of vertebral compression fractures. BMC Surg 14: 3, 2014. 
27. Voggenreiter G: Balloon kyphoplasty is effective in deformity correction of osteoporotic vertebral compression fractures. Spine 30: 2806-2812, 2005.

28. Ledlie JT and Renfro MB: Kyphoplasty treatment of vertebral fractures: 2-year outcomes show sustained benefits. Spine 31: 57-64, 2006.

29. Phillips FM, Ho E, Campbell-Hupp M, McNally T, Todd Wetzel F and Gupta P: Early radiographic and clinical results of balloon kyphoplasty for the treatment of osteoporotic vertebral compression fractures. Spine 28: 2260-2267, 2003.

30. Fourney DR, Schomer DF, Nader R, Chlan-Fourney J, Suki D, Ahrar K, Rhines LD and Gokaslan ZL: Percutaneous vertebroplasty and kyphoplasty for painful vertebral body fractures in cancer patients. J Neurosurg 98 (Suppl): 21-30, 2003.

31. Pflugmacher R, Schleicher P, Schröder RJ, Melcher I and Klostermann CK: Maintained pain reduction in five patients with multiple myeloma 12 months after treatment of the involved cervical vertebrae with vertebroplasty. Acta Radiol 47: 823-829, 2006.
32. Eck JC, Nachtigall D, Humphreys SC and Hodges SD: Comparison of vertebroplasty and balloon kyphoplasty for treatment of vertebral compression fractures: A meta-analysis of the literature. Spine J 8: 488-497, 2008

33. Cotten A, Boutry N, Cortet B, Assaker R, Demondion X, Leblond D, Chastanet P, Duquesnoy B and Deramond H: Percutaneous vertebroplasty: State of the art. Radiographics 18: 311-323, 1998.

34. Deramond H, Depriester C, Galibert P and Le Gars D: Percutaneous vertebroplasty with polymethylmethacrylate. Technique, indications, and results. Radiol Clin North Am 36: 533-546, 1998.

35. Garfin SR, Yuan HA and Reiley MA: New technologies in spine: Kyphoplasty and vertebroplasty for the treatment of painful osteoporotic compression fractures. Spine 26: $1511-1515,2001$ 